\begin{tabular}{|c|l|}
\hline Title & Signal propagation and failure in one dimensional FitzH ugh-Nagumo equations with periodic stimuli \\
\hline Author(s) & Y anagita, T.; Nishiura, Y asumasa; Kobay ashi, R. \\
\hline Citation & $\begin{array}{l}\text { Physical Review E, 71,036226 } \\
\text { https://doi.org/10.1103/PhysRevE.71.036226 }\end{array}$ \\
\hline Issue Date & 2005-03-30 \\
\hline Doc URL & http://hdl.handle.net/2115/5427 \\
\hline Rights & Copyright $\odot 2005$ A merican Physical Society \\
\hline Type & article \\
\hline File Information & PRE71-3.pdf \\
\hline
\end{tabular}

Instructions for use 


\title{
Signal propagation and failure in one-dimensional FitzHugh-Nagumo equations with periodic stimuli
}

\author{
T. Yanagita* and Y. Nishiura ${ }^{\dagger}$ \\ Laboratory of Nonlinear Studies and Computation, Research Institute for Electronic Science, Hokkaido University, \\ Sapporo 060-0812, Japan \\ R. Kobayashi ${ }^{\ddagger}$ \\ Department of Mathematical and Life Sciences, Hiroshima University, Higashi-Hiroshima, 739-8626, Japan
}

(Received 10 August 2004; published 30 March 2005)

\begin{abstract}
We analyze the effect of additive periodic stimuli in one-dimensional FitzHugh-Nagumo equations in an excitable regime. With a suitable stimulus interval, the suppression of the pulse propagation occurs in some parameter regime. This propagation failure comes from the formation of the "death spot" where successive pulses annihilate. In the parameter regime where the solitary pulse cannot propagate in space stably, however, periodic stimuli cause a propagation of envelope of a traveling pulse under a "resonance" condition, i.e., the pulse at the leading edge disappears successively, however, an envelope is formed and propagates with keeping its shape.

\section{DOI: 10.1103/PhysRevE.71.036226}

Numerous chemical [1,2] and biological [3] phenomena

PACS number(s): 82.40.Bj, 05.45.-a

$$
v_{t}=u-\gamma v, \quad x \in(0, L),
$$
\end{abstract}
can be modeled in the framework of reaction-diffusion systems, which have revealed an unexpectedly rich variety of dynamical behaviors of pulses [4-6]. Excitable systems play a fundamental role in neural information processing and many other biological systems. An initial stimulus is needed to generate pulse propagation in an excitable regime, and the inclusion of repetitive stimuli shows a variety of response patterns [7-9]. Often, one measures only large amplitude suprathreshold activity at a particular location in the system, for instance, the action potential in a nerve or ventricular contraction in heart. An interesting feature of such an activity is that in response to a periodic stimuli, the times between these large excursion events can, when plotted in interspike interval histograms [10], produce multimodal peaks. A recent hypothesis is that such distributions may be associated with stochastic resonance $[11,12]$. On the contrary, the possible contribution of chaotic dynamics to the irregular firing is considered $[13,14]$.

The single element models are, however, too simple to allow the activity on an actual neuron to be studied. Most neurons have complicated shapes, particularly bifurcation patterns in dendrites and axon terminals. Thus consideration of the spatial degree of freedom is needed. It is known that a localized stimulus of finite amplitude forms a propagating pulse for spatially extended excitable systems, however, the effect of periodic stimuli incorporating the spatially extended system is not well known.

Here, we demonstrate the effect of periodic stimuli on spatiotemporal pattern in one-dimensional FitzHughNagumo (FHN) model $[15,16]$ with no noise,

$$
\tau u_{t}=u(u-\alpha)(1-u)-v+\epsilon^{2} u_{x x},
$$

\footnotetext{
*Electronic address: yanagita@nsc.es.hokudai.ac.jp †Electronic address: nishiura@aurora.es.hokudai.ac.jp

*Electronic address: ryo@math.sci.hiroshima-u.ac.jp
}

where $\alpha, \epsilon, \gamma$, and $\tau$ are parameters, $u=u(x, t)$ the activator and $v=v(x, t)$ the inhibitor.

A homogeneous initial condition $u(x, 0)=v(x, 0)=0$ is a global stable solution with Neumann boundary condition $\partial u /\left.\partial x\right|_{x=0}=\partial u /\left.\partial x\right|_{x=L}=0$. Successive stimuli with a constant time interval $t_{s}$ are applied instantaneously in the region $x \in[0, l]$ periodically for a small $l$, i.e., $u(x, t)=u_{0}$ for $x$ $\in[0, l]$ and $t=\left\{t \mid n t_{s}, n \in \mathcal{N}\right\}$ where $\delta(x)$ is delta function.

Employing the following parameters: $\alpha=0.0, \epsilon=0.05, \tau$ $=0.018, \gamma=1.0, L=20, l=0.2, u_{0}=0.5$, all pulses generated by each stimulus propagate in space for large $t_{s}$ say $t_{s}=10.0$. The parameter values used here admit the "oscillating wake" of traveling pulse since the eigenvalues of the uniform stationary solution $(u, v)=(0,0)$, which are given by $\lambda_{ \pm}=[-\alpha$ $\left.-\gamma \tau \pm \sqrt{-4(1+\alpha \gamma) \tau+(\alpha+\gamma \tau)^{2}}\right] /(2 \tau)$, have an imaginary part. Then the intrinsic period of the oscillation that is given by $t_{\lambda}=2 \pi /|\operatorname{Im}(\lambda)|$ where Im represents imaginary part. In Fig. 1 , the spatial pattern and phase plane plot of solitary traveling pulse with both monotonic and oscillating wake are depicted. The time evolution of $u, v$ for $x=l / 2$, the center of the stimulation region, after a single stimulus at $t=0$ is shown in Fig. 2. Due to the oscillation, the excitability of the system varies in time. The light and dark gray regions depicted in Fig. 2 indicate the time intervals where excitable period $[u(l / 2, t)>0] \wedge[v(l / 2, t)<0]$ and refractory period $v(l / 2, t)$ $>0$, respectively. For the light gray intervals, a relatively small stimulus can excite the system, however, the system cannot respond to any stimulus for the dark intervals.

When $\tau$ is changed to 0.019 , the solitary pulse generated by the single stimulus at $t=0$ with $\left(l, u_{0}\right)$ where $\{l \mid 0.02,0.05,0.1,0.2\}$ and $\left\{u_{0} \mid 0.5,1.0,1.5,2.0\right\}$ disappears eventually after traveling a finite distance. In order to determine the critical value numerically, we examine the norm, $n(t)=\int_{0}^{L} u^{2}(x, t)+v^{2}(x, t) d x$, of the solitary pulse, which converges to a positive value as $t$ increases for a sufficiently 

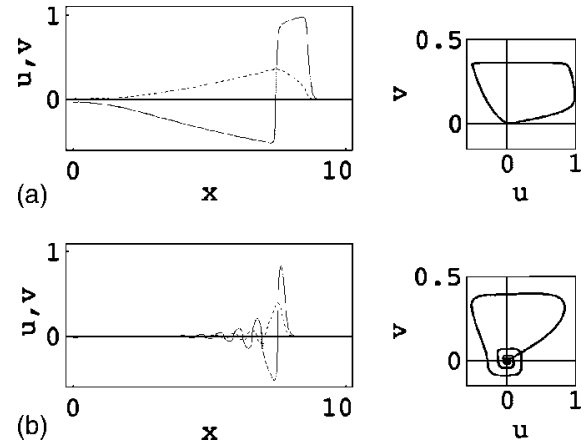

FIG. 1. Spatial pattern and phase plane plot are shown for both monotonic and oscillating wake pulse. To see the tiny oscillating trail, we have used the transformation $f(x)=\operatorname{sgn}(x)|x|^{1 / 2}$ for $u$ and $v$. In spatial patterns (left side), the full (dotted) line indicates the profile of $f(u)[f(v)]$. (a) Monotonic wake pulse: $\alpha=0.1, \epsilon$ $=0.05, \gamma=1.0, \tau=0.002$. (b) Oscillating wake pulse: $\alpha=0.0, \epsilon$ $=0.05, \gamma=1.0, \tau=0.0185$.

large system size when the solitary pulse propagates stably. By gradually increasing the parameter $\tau$ from 0.018 to 0.019 , we have found a critical value $\tau=\tau_{c} \sim 0.01876$ above which the norm converges to zero and the solitary pulse can propagate transiently for some finite interval [19].

Periodic stimuli form a pulse train whose spatiotemporal behavior depends on the parameter $t_{s}$ and $\tau$. Near the critical parameter, we have found the following phenomena: (a) propagation failure, the leading pulse of the train vanishes successively at some position after traveling a finite distance even if the solitary pulse can propagate stably; (b) resonance behavior, the pulse train can gradually propagate in space with a suitable $t_{s}$ although the solitary pulse disappears after traveling a small distance. These exotic things depend in a subtle way on the ratio between external period $t_{s}$ and the intrinsic period of the system as explained in the following. The term "resonance" stems from these two time scales. Note that the traveling pulse solution without oscillating wake does not show these exotic behaviors. Phase locking is similarly observed for shorter $t_{s}$, however, the propagation failure does not occur; the profile of stimulus response ratio (SRR) is equal to 1.0 for $t_{s}>1.0$. When the solitary pulse solution disappears through saddle-node bifurcation, any stimulus interval cannot show resonance behavior; the SRR

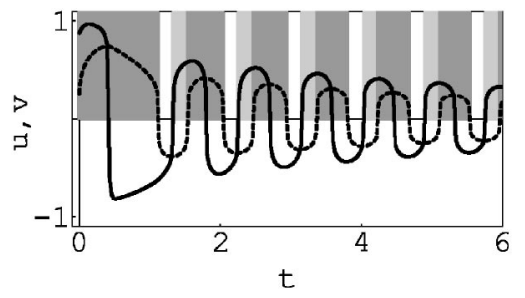

FIG. 2. The time evolution of $u(x, t)$ and $v(x, t)$ at the middle of the stimulation area $x=l / 2$, after a single stimulus, $\tau=0.01850$. The light and the dark gray regions represent the time intervals where $[u(t)>0] \wedge[v(t)<0]$ and $v(t)>0$, respectively. The light gray region is easy to fire by the tiny stimulus, and the dark gray region corresponds to refractory period. These time intervals are also shown in Fig. 4. equal to 0 for all $t_{s}$. Furthermore, constant stimulus, Dirichlet boundary condition at $u(0, t)=$ const, for example, cannot produce pulse repeatedly, and thus these exotic phenomena are never observed. The failure and resonance originate in both the repetition of stimulation and oscillating wake of the pulse.

Typical spatiotemporal patterns, locking, chaotic, propagation failure, and resonance are shown in Fig. 3. To characterize these response behaviors, it has been used the stimulus-response ratio (SRR) [7-9]. For spatially extended system, we introduce SRR as the ratio of the number of the pulses that reach the opposite boundary $x=L$ to the number of the stimuli as depicted in Fig. 4 for a sufficiently large $L$. In the following, we explain each spatiotemporal behavior together with the SRR.

When the stable solitary traveling pulse exists, every pulse generated by the stimulus would reach the opposite boundary for larger $t_{s}$. Thus the SRR is equal to 1 , that is each stimulus would be followed by one traveling pulse, as it is clearly shown in Fig. 4(a). However, the pulse propagation does not occur by the stimulus with a shorter interval since there are time intervals during which a perturbation does not induce a new excitation. This interval is called the refractory period. Since the refractory period varies in time attributable to the oscillating wake, as shown in Fig. 2, whether the pulse train generated by periodic stimuli propagates stably depends on $t_{s}$ relative to the intrinsic period $t_{\lambda}$.

Around $t_{s} \sim 0.6$ and 2.0 in Fig. 4(a), the pulse generated every other stimuli and its spatiotemporal plot is also shown in Fig. 3(a). The plateau of SRR shows phase locking or parameter-response locking observed in many of chaotic systems $[9,17]$. Chaotic behavior is also observed at intermediate values between two locking state. Pulses among the pulse train irregularly vanish [Fig. 3(b)]. Interspike interval histogram in the chaotic region is multimodal. The multimodal distribution is also obtained by the FHN model without spatial degree of freedom [13]. We note that the parameters used in Ref. [13] also admit an oscillating wake since the eigenvalues of the rest state have imaginary parts.

For some parameter regions in which the SRR is 0 in Fig. $4(\mathrm{a})$, for example, $t_{s} \in[2.82,2.87]$, the pulse train can propagate for some finite interval and cannot reach the opposite boundary $x=L$ despite the existence of stable solitary pulse. This propagation failure is caused by the formation of the "death spot" where successive pulses annihilate suddenly after an initial transient. The spatiotemporal pattern for the propagation failure is depicted in Fig. 3(c). The death spot is formed by the following manner: once the pulse disappears at a certain position by the effect of the oscillating wake of the preceding pulse, the refractory period around the disappeared position (death spot) varies in time because of the after effect of the annihilation. When the time at which the following pulse arrived at the death spot is a refractory period, the pulse vanishes almost at the same position. After the repetition of the annihilation process, the death spot is synchronized with external period $t_{s}$ by adjusting its position. Finally, the stationary death spot is formed at a certain location. After forming the death spot, all pulses generated by periodic stimuli propagate in space, and they reach the death spot and then vanish [see Fig. 3(c)]. 

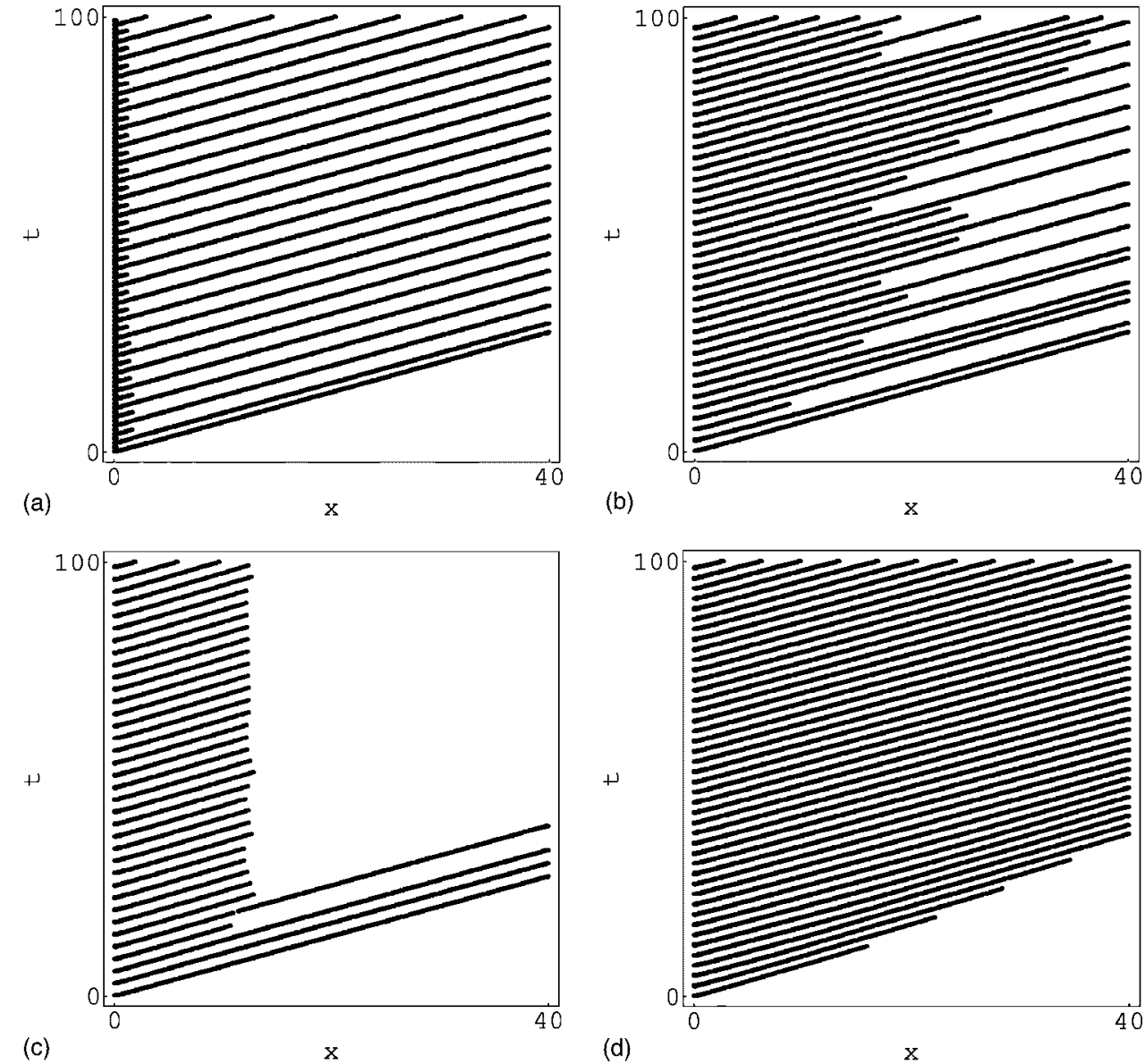

FIG. 3. The spatiotemporal patterns of periodically stimulated FHN equations. A stable traveling pulse exists in the following parameters $\tau=0.0185, \epsilon=0.05, \alpha=0.0, \gamma=1.0, l=0.2$ (a)-(c). (a) $t_{s}=1.0$ : the pulse generated every other stimulus; (b) $t_{s}=2.5$ : the pulse generates chaotically. (c) $t_{s}=2.82$ : The propagation failure of traveling pulse. The pulses cannot propagate in space. Note that the stable traveling pulse exists in this parameter region. (d) $t_{s}=2.34$ : the solitary pulse propagates to a certain location and annihilates for $\tau=0.01885$. Each pulse annihilates, however, the position of the leading pulse of the train gradually increase in time.
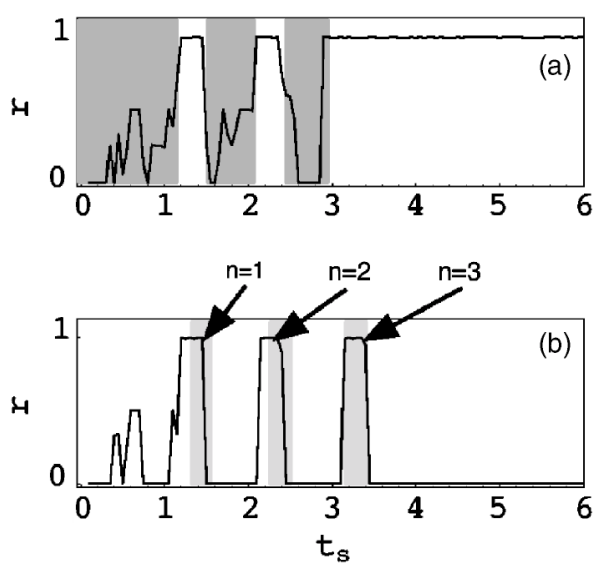

FIG. 4. The stimulus response ratio $r$ is calculated by changing the stimulus interval $t_{s}$. (a) $\tau=0.018$ 50: the zero firing rate near $t_{s}$ $=0.4$ and 0.75 correspond to propagation failure in Fig. 3(c). (b) $\tau$ $=0.018$ 85: indicated number is the order of the resonance plateau. The light and the dark gray regions show excitable and refractory period estimated from the time evolution as shown in Fig. 2, respectively.
Above $\tau_{c}$, it is observed that the stable solitary pulse does not exist, and the homogeneous state $u(x)=v(x)=0$ is globally stable in the absence of the repetitive stimulus. In this parameter region, the solitary pulse generated by the single stimulus at $t=0$ propagates transiently for some finite interval and disappears. By adding periodic stimuli, short lifetime pulses are generated successively for a larger $t_{s}$ since the preceding pulse cannot affect the following pulse. However, as we see in Fig. 3(d), the formation of traveling pulse train is observed with a suitable forcing period. Since the solitary pulse cannot propagate stably in the parameter regime, the leading pulse of the train disappears, while the envelope of these waves expands as a function of time.

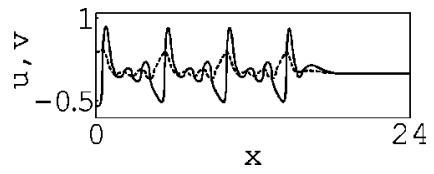

FIG. 5. The spatial pattern where resonance occurred $(\tau$ $=0.1885$ and $t_{s}=3.33$ ). The solitary pulse cannot propagate stably in this parameter region. The full (dotted) line indicates the profile of $f(u)[f(v)]$ where $f(x)$ is same as in Fig. 1. 


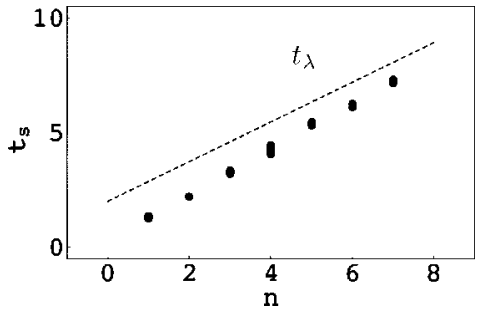

FIG. 6. The order of the resonance region versus stimulus time interval $t_{s}$ is plotted. Stimulus time intervals which cause resonance fitted to $t_{s} \sim t_{\lambda} n$ (broken line) asymptotically for larger $n ; \tau$ $=0.01884$.

The heuristic reason for this behavior lies in the following fact: imaginary part of eigenvalues makes oscillating wake behind the traveling pulse (see Fig. 5, the snapshot of the profile of $u, v$ is plotted where the oscillating wake is clearly shown). If the timing of the stimulus coincides with the bump of the oscillating wake for the preceding pulse, i.e., light gray time intervals in Fig. 2, the "resonance condition" is satisfied and the front pulse preserves the following pulse which is generated by the next stimulus.

The time interval where the SRR value equals 1 will be called the resonance region that is characterized by the intrinsic period $t_{\lambda}$ in the following. We number each of the plateaus where the SRR value is larger than 0.99 in the increasing order from $t_{s}=0$ and plot the order $n$ of the plateau versus stimulus time interval $t_{s}$ in Fig. 6 . Near the critical parameter $\tau \sim \tau_{c}$, the relation $t_{s}(n) \sim t_{\lambda} n$ holds for larger $n$ that means resonance structures appear periodically with the interval $t_{\lambda}$.

The number of resonance areas depends on the distance to the critical point. In fact, if one chooses $\tau$ close to the critical value $\tau_{c}$ (saddle-node bifurcation point), the number of the resonance areas will be increased. In Fig. 7, the dependence of $t_{s}$ and $\tau$ on SRR is depicted, where the resonance structure emerges at a larger stimulus interval near the critical point.

In summary, we have investigated the propagation failure and the resonance behavior in Eqs. (2) with periodic stimuli

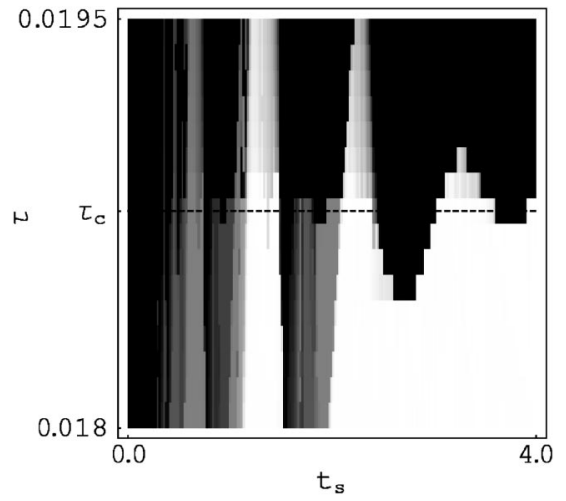

FIG. 7. The parameter $\tau$ and stimulus interval $t_{s}$ dependency of the SRR shown as a density plot, where black (white) is 0 (1), and gray is an intermediate SRR value between 0 and 1 . The broken line indicates $\tau=\tau_{c} \sim 0.18838$ where the solitary traveling solution disappears. Above the critical value, the solitary traveling pulse cannot propagate stably.

for a simple excitable parameter region. An essential dynamical ingredient of the behavior is that the solitary traveling pulse has an oscillating wake, which comes from the existence of the imaginary part of eigenvalues for the stationary solution. These phenomena can be observed in any excitable media near the critical point where the stable solitary traveling pulse with oscillating wake disappears through saddle-node bifurcation. It is also interesting to contemplate the infinitely long pulse train in $\mathcal{R}^{1}$ which has the leading pulse. Here, the boundary between the region with and without the pulse train would be move forward (the same direction of the pulse motion) or backward depending on the parameters. The study of these dynamics is of general application to the understanding of disordered phenomena in spatially extended excitable media, and may provide interesting insight about excitable systems such as the cardiac dynamics.

This work was partially supported by Grant-in-Aids for Scientific Research from the Ministry of Education, Science, and Culture of Japan.
[1] A. N. Zaikin and A. M. Zhabotinsky, Nature (London) 255, 535 (1970)

[2] A. T. Winfree, Science 175, 634 (1972).

[3] S. Kondo and R. Asai, Nature (London) 376, 765 (2002).

[4] Y. Hayase and T. Ohta, Phys. Rev. E 66, 036218 (2002).

[5] R. Kobayashi, T. Ohta, and Y. Hayase, Phys. Rev. E 50, R3291 (1994).

[6] G. V. Osipov and J. J. Collins, Phys. Rev. E 60, 54 (1999).

[7] R. D. Chialvo and J. Jalife, Nature (London) 330, 749 (1987).

[8] L. Glass, Nature (London) 410, 277 (2001).

[9] G. Matsumoto, K. Aihara, Y. Hanyu, and N. Takahashi, Phys. Lett. A 123, 162 (1987).

[10] R. M. Siegel, Physica D 42, 385 (1990).

[11] K. Weisenfeld and F. Moss, Nature (London) 373, 33 (1995).

[12] V. A. Makarov, V. I. Nekorkin, and M. G. Velarde, Phys. Rev. Lett. 86, 3431 (2001).
[13] D. T. Kaplan, J. R. Clay, T. Manning, L. Glass, M. R. Guevara, and A. Shrier, Phys. Rev. Lett. 76, 4074 (1996).

[14] J. R. Clay, Phys. Rev. Lett. 76, 4074 (1996).

[15] R. FitzHugh, Biophys. J. 1, 445 (1961).

[16] J. S. Nagumo, S. Arimoto, and S. Yoshizawa, Proc. IRE 50, 2061 (1962).

[17] L. Glass and R. Perez, Phys. Rev. Lett. 48, 1772 (1982).

[18] E. J. Doedel, A. R. Champneys, T. F. Fairgrieve, Y. A. Kuznetsov, B. Sandstede, and X. Wang, ftp://ftp.cs.concordia.ca/ pub/doedel/auto

[19] We have also analyzed "transient pulse" which disappear eventually after traveling a finite distance with different parameter regime. Bifurcation analysis by using AUTO software [18] showed that the solitary traveling pulse solution disappears through saddle-node bifurcation. Here, we expect the solitary pulse disappear through saddle-node bifurcation at $\tau=\tau_{c}$. 